Received: 14 March 2017

Accepted: 18 October 2017

Published online: 08 November 2017

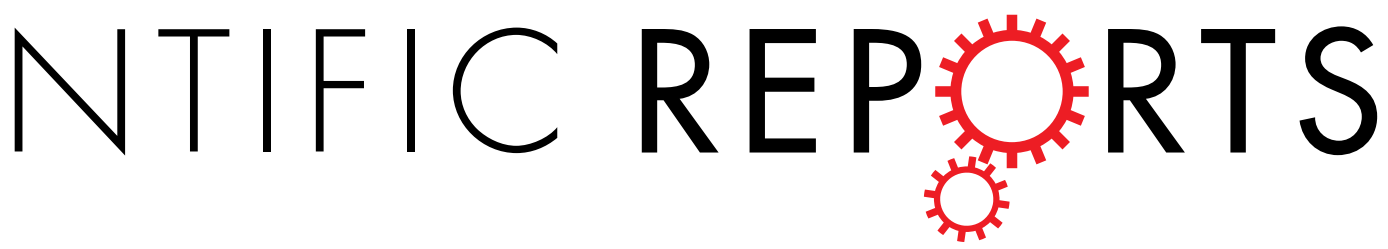

\title{
OPEN Temporal and spatial expression of genes involved in DNA methylation during reproductive development of sexual and apomictic Eragrostis curvula
}

\author{
J. P. Selva ${ }^{1,2}$, L. Siena ${ }^{3}$, J. M. Rodrigo ${ }^{1,4}$, I. Garbus ${ }^{5}$, D. Zappacosta ${ }^{1,4}$, J. R. Romero ${ }^{1}$, \\ J. P. A. Ortiz ${ }^{3}$, S. C. Pessino ${ }^{3}$, O. Leblanc ${ }^{6} \&$ V. Echenique ${ }^{1,4}$
}

Recent reports in model plant species have highlighted a role for DNA methylation pathways in the regulation of the somatic-to-reproductive transition in the ovule, suggesting that apomixis (asexual reproduction through seeds) likely relies on RdDM downregulation. Our aim was therefore to explore this hypothesis by characterizing genes involved in DNA methylation in the apomictic grass Eragrostis curvula. We explored floral transcriptomes to identify homologs of three candidate genes, for which mutations in Arabidopsis and maize mimic apomixis (AtAG09/ZmAG0104, AtCMT3/ZmDMT102) ZmDMT105, and AtDDM1/ZmCHR106), and compared both their spatial and temporal expression patterns during reproduction in sexual and apomictic genotypes. Quantitative expression analyses revealed contrasting expression patterns for the three genes in apomictic vs sexual plants. In situ hybridization corroborated these results for two candidates, ECAG0104 and ECDMT102, and revealed an unexpected ectopic pattern for the $A G O$ gene during germ line differentiation in apomicts. Although our data partially support previous results obtained in sexual plant models, they suggest that rather than an RdDM breakdown in the ovule, altered localization of AtAG09/ZmAG0104 expression is required for achieving diplospory in $E$. curvula. The differences in the RdDM machinery acquired during plant evolution might have promoted the emergence of the numerous apomictic paths observed in plants.

Unlike the genetically diverse progeny produced by sexual reproduction in flowering plants, the asexual reproductive process of apomixis results in seeds containing maternal clones ${ }^{1}$. Apomixis is widely distributed among angiosperms ${ }^{2}$, and its emergence is thought to have required a deregulation of both the genetic and epigenetic components of female reproduction in sexual ancestors ${ }^{3}$.

Seed formation in sexual plants is achieved through a double fertilization event after the encounter of the female gametophyte with the male gametophyte, both carrying two gametes. Gametophytes typically differentiate from meiotic products, the spores. Female meiosis initiates within a single cell, specified in the nucellus of the ovule, the megaspore mother cell (MMC). Upon meiosis the MMC produces a tetrad of reduced cells, out of which a single functional product, the megaspore (MS), undergoes mitotic divisions and differentiation. The resulting gametophyte, or the embryo sac (ES), is seven-celled at maturity and contains two female gametes, the egg cell and the binucleate central cell ${ }^{4}$. During the double fertilization process, each female gamete fuses with one of the two sperm cells delivered by the pollen grain (the male gametophyte). This triggers the formation of

${ }^{1}$ CERZOS-CONICET, CCT-Bahía Blanca, 8000, Bahía Blanca, Argentina. ${ }^{2}$ Departamento de Biología, Bioquímica y Farmacia, Universidad Nacional del Sur, 8000, Bahía Blanca, Argentina. ${ }^{3}$ IICAR-CONICET/Laboratorio de Biología Molecular, Facultad de Ciencias Agrarias, Universidad Nacional de Rosario, Parque Villarino, S2125ZAA, Zavalla, Argentina. ${ }^{4}$ Departamento de Agronomía, Universidad Nacional del Sur, 8000, Bahía Blanca, Argentina. ${ }^{5}$ Departamento de Ciencias de la Salud, Universidad Nacional del Sur, 8000, Bahía Blanca, Argentina. ${ }^{6}$ DIADE, IRD, Univ Montpellier, Montpellier, France. J.R. Romero is deceased. Correspondence and requests for materials should be addressed to O.L. (email: olivier.leblanc@ird.fr) or V.E. (email: echeniq@criba.edu.ar) 
the embryo and the endosperm, respectively, and initiates seed development with the two fertilization products embedded in maternal tissues.

Apomixis refers to alternative reproductive pathways that allow somatic (i.e., unreduced) cells within the ovule to acquire a reproductive fate after the triggering of either embryogenesis (adventitious embryony) or female gametogenesis in the absence of meiosis followed by autonomous egg cell development (gametophytic apomixis $)^{5}$. Gametophytic apomixis involves a broad spectrum of developmental pathways and is sub-categorized into either (1) diplospory, where the unreduced ES, derives from a MMC that fails to achieve meiosis and initiates gametogenesis, or (2) apospory, when several nucellar or integumental cells differentiate directly into unreduced ESs. In all gametophytic apomicts, seed development requires endosperm formation either autonomously or after central cell fertilization (pseudogamy) ${ }^{5}$.

Gametophytic apomixis is controlled genetically by a few undetermined factors ${ }^{6}$. Recent functional studies highlighted a role for small interfering RNAs (siRNAs) in apomictic pathways, as the depletion of an RNA-directed DNA methylation (RdDM) pathway specifically acting in plant ovules promotes an asexual reproductive fate ${ }^{3}$. This observation is supported by transcriptomic analyses in several species, e.g. Boechera gunnisoniana ${ }^{7}$, Hypericum perforatum ${ }^{8,9}$ and Hieracium spp. ${ }^{10}$. Members of the ARGONAUTE4 clade in Arabidopsis thaliana (AGO4, AGO6, AGO8 and AGO9) encode proteins binding heterochromatic siRNAs that target sequence repeats and transposable elements, and mutations in these genes result in the formation of extra female gametophytic precursors, suggesting they play a role in the somatic-to-reproductive transition in ovules ${ }^{11,12}$. In maize (Zea mays), loss-of-function of AGO104, a homolog of Arabidopsis AGO9, results in functional unreduced female gametes arising from mitosis-like divisions in the MMC, but without affecting the number of germ cells ${ }^{13}$. AtAGO9 and $\mathrm{ZmAGO104}$ therefore seem to promote different functions during reproduction in Arabidopsis and maize, respectively repressing the germ cell fate of somatic cells and the somatic cell fate in the germ line ${ }^{3}$. This difference likely explains the phenotypic consequences of AtAGO9/ZmAGO104 depletion, which mimics apospory in Arabidopsis and diplospory in maize.

It was reported that after the deregulation of several other genes involved in DNA methylation pathways apomixis-like phenotypes were observed in model plant species ${ }^{11,14}$. These genes include: 1) the sequences encoding two Arabidopsis DNA methyltransferases (DMTs) CHROMOMETHYLTRANSFERASE3 (CMT3) and DOMAIN REARRANGED METHYLTRANSFERASE1 (DRM1), as well as their maize respective homologs ZmDMT102/ZmDMT105 and ZmDMT103; 2) the Arabidopsis DECREASE IN DNA METHYLATION1 (DDM1) chromatin-remodeling factor and its maize homolog ZmCHR106; and 3) the Arabidopsis sequences regulating small interfering RNAs biogenesis RNA-DEPENDENT RNA POLYMERASE2 (RDR2), DICER-LIKE3 (DCL3) and DNA-DIRECTED RNA POLYMERASES $V$ and $I V$. Other than RT-PCR analyses in diplosporous wild relatives of Arabidopsis and maize, (Boechera holboellii and Tripsacum dactyloides, respectively) ${ }^{14}$, no studies have investigated the role of specific epigenetic pathways in natural apomicts.

Eragrostis curvula (weeping lovegrass) is a perennial grass that reproduces mainly by diplosporous apomixis, although fully sexual plants occur sporadically ${ }^{15,16}$. The use of this model system allowed us to test for a link between the deregulation of DNA methylation pathways and the occurrence of apomixis in a natural grass system. To this end, we identified the homologs of AtAGO9/ZmAGO104, ZmDMT102 and ZmCHR106 using E. curvula floral transcriptomes and showed they were differentially expressed in the ovules of sexual and apomictic plants. Our results support the findings of previous functional analyses, performed mainly in sexual model plant species, for an essential role of the RdDM pathway in the switch from sexual to diplosporous development.

\section{Results}

Transcript identification in sexual and apomictic $E$. curvula transcriptome databases. Recently we reported an E. curvula reference transcriptome ${ }^{17}$ that constitutes an important step toward the identification of genes controlling key steps of the apomictic pathway. Roche 454 sequencing technology was used to generate reads from inflorescences of E. curvula apomictic and sexual genotypes. The resulting reads were de novo assembled using the Newbler Assembler software v2.6 (Roche, Indianapolis, IN, USA) into 49568 isotigs that were further grouped into 25186 isogroups. Near $90 \%$ of the unigenes showed high similarity to sequences from public databases.

To identify the E.curvula orthologs of the three candidate genes we selected, protein sequences of the maize $\mathrm{AGO}^{18}, \mathrm{DMT}^{19}$, and $\mathrm{CHR}^{14}$ families were queried against the E. curvula reference transcriptome. High levels of sequence similarity were found for most queries, including 11 out of 17 AGOs, five of the eight DMTs, and all eight CHR members (Tables 1, 2 and 3, respectively).

To support these results, we next generated maximum likelihood phylogenetic trees for the AGO, DMT, and CHR families using protein sequences from maize, Arabidopsis and Eragrostis. As shown in Fig. 1, the clustering of homologs confirmed relatedness between Eragrostis sequences and that whose loss of function leads to reproductive behaviors reminiscent of apomixis in Arabidopsis and maize. Thus, we renamed isotig33988 as EcAGO104, isotig25116 as EcDMT102 and isotig25194 as EcCHR106.

Quantitative expression analysis. We first compared the expression profiles of EcAGO104, EcDMT102 and $E c C H R 106$ using the 454-sequencing data derived from the sexual and apomictic libraries ${ }^{17}$. Differential expression analysis using the EdgeR package detected a significant difference only for EcAGO104 that showed higher expression in apomictic plants compared to sexual counterparts ( $\mathrm{p}$ value $=1.17 \mathrm{E}-05$ ). These results are discordant with previous reports indicating downregulation for all three genes in apomict plants ${ }^{15,16}$. However, since our reference transcriptome was generated using a mix of all reproductive developmental stages, it is possible that we could not detect more subtle transcriptional differences using this approach. Therefore, we performed quantitative RT-PCR using mRNAs extracted from flowers at both archesporial and gametophytic stages to compare transcript abundances between sexual and apomictic plants (Fig. 2). No significant difference in expression 


\begin{tabular}{|l|l|l|l|l|l|}
\hline Query $^{\mathbf{a}}$ & Subject (E. curvula) & \% identity & Alignment length & e-value & Score \\
\hline ZmAGO1a & N/A & N/A & N/A & N/A & N/A \\
\hline \multirow{2}{*}{ ZmAGO1b } & isotig10363 & 93.98 & 1079 & 0 & 2047 \\
\cline { 2 - 6 } & isotig09228 & 87.81 & 1124 & 0 & 1949 \\
\hline ZmAGO1c & N/A & N/A & N/A & N/A & N/A \\
\hline \multirow{2}{*}{ ZmAGO1f } & isotig33848 & 84.75 & 1056 & 0 & 1755 \\
\cline { 2 - 6 } & isotig18801 & 78.36 & 1049 & 0 & 1593 \\
\hline ZmAGO2a & N/A & N/A & N/A & N/A & N/A \\
\hline ZmAGO2b & isotig33909 & 72.96 & 858 & 0 & 1228 \\
\hline \multirow{2}{*}{ ZmAGO4 } & isotig12569 & 87.06 & 904 & 0 & 1598 \\
\cline { 2 - 6 } & isotig25978 & 61.94 & 867 & 0 & 1061 \\
\hline ZmAGO5a & isotig03447 & 70.91 & 495 & 0 & 699 \\
\hline ZmAGO5b & isotig18741 & 81.56 & 526 & 0 & 869 \\
\hline ZmAGO5c & isotig25155 & 86.95 & 659 & 0 & 1145 \\
\hline ZmAGO5d & N/A & N/A & N/A & N/A & N/A \\
\hline ZmAGO7 & isotig38102 & 86.17 & 412 & 0 & 739 \\
\hline ZmAGO9 & isotig33988 & 86.51 & 912 & 0 & 1622 \\
\hline ZmAGO10a & N/A & N/A & N/A & N/A & N/A \\
\hline ZmAGO10b & isotig33998 & 96.23 & 664 & 0 & 1316 \\
\hline ZmAGO18a & isotig34174 & 68.44 & 716 & 0 & 968 \\
\hline ZmAGO18b & N/A & N/A & N/A & N/A & N/A \\
\hline
\end{tabular}

Table 1. Members of the ARGONAUTE family queried against the Eragrostis curvula protein prediction databases. ${ }^{a}$ Query names as described by ${ }^{18}$. They renamed ZmAGO104 to ZmAGO9; therefore, the isotig33988 is the ortholog of ZmAGO104.

\begin{tabular}{|l|l|l|l|l|l|}
\hline Query $^{\mathrm{a}}$ & Subject $($ E. curvula) & \% identity & Alignment length & e-value & Score \\
\hline \multirow{2}{*}{ ZmMET1a } & isotig33722 & 87.61 & 702 & 0 & 1289 \\
\cline { 2 - 7 } & isotig33939 & 70.82 & 682 & 0 & 1026 \\
\hline ZmMET1b & N/A & N/A & N/A & N/A & N/A \\
\hline ZmMET2a (ZmDMT102/105) & isotig25116 & 69.84 & 610 & 0 & 866 \\
\hline ZmMET2b & N/A & N/A & N/A & N/A & N/A \\
\hline ZmMET3a & isotig22920 & 81.68 & 382 & 0 & 662 \\
\hline ZmMET3b & N/A & N/A & N/A & N/A & N/A \\
\hline ZmMET3c & isotig25609 & 72.21 & 457 & 0 & 642 \\
\hline ZmMET4 & isotig27287 & 84.64 & 358 & 0 & 619 \\
\hline
\end{tabular}

Table 2. Members of the DNA methyltransferase family queried against the Eragrostis curvula protein prediction databases. ${ }^{\mathrm{a}}$ Query names as described by ${ }^{19}$. They renamed ZmDMT102 as ZmMET2a; therefore, isotig25117 is the ortholog of ZmDMT102.

\begin{tabular}{|l|l|l|l|l|l|}
\hline Query $^{\text {a }}$ & Subject (E. curvula) & \% identity & Alignment length & e-value & Score \\
\hline ZmCHR125 & isotig16268 & 85.32 & 1260 & 0 & 2145 \\
\hline ZmCHR113 & isotig13154 & 81.61 & 1338 & 0 & 2186 \\
\hline ZmCHR120 & isotig08900 & 63.36 & 1261 & 0 & 1474 \\
\hline ZmCHR126 & isotig02865 & 93.11 & 1118 & 0 & 2051 \\
\hline ZmCHR106 & isotig25194 & 85.59 & 708 & 0 & 1204 \\
\hline ZmCHR101 & isotig25194 & 84.97 & 712 & 0 & 1211 \\
\hline \multirow{2}{*}{ ZmCHR112 } & isotig34902 & 61.25 & 542 & 0 & 679 \\
\cline { 2 - 6 } & isotig37943 & 81.98 & 394 & 0 & 660 \\
\hline ZmCHR110 & isotig02865 & 93 & 928 & 0 & 1718 \\
\hline
\end{tabular}

Table 3. Members of the SNF2 super family queried against the Eragrostis curvula protein prediction databases. ${ }^{\mathrm{a}}$ Query names as described by ${ }^{14}$. 

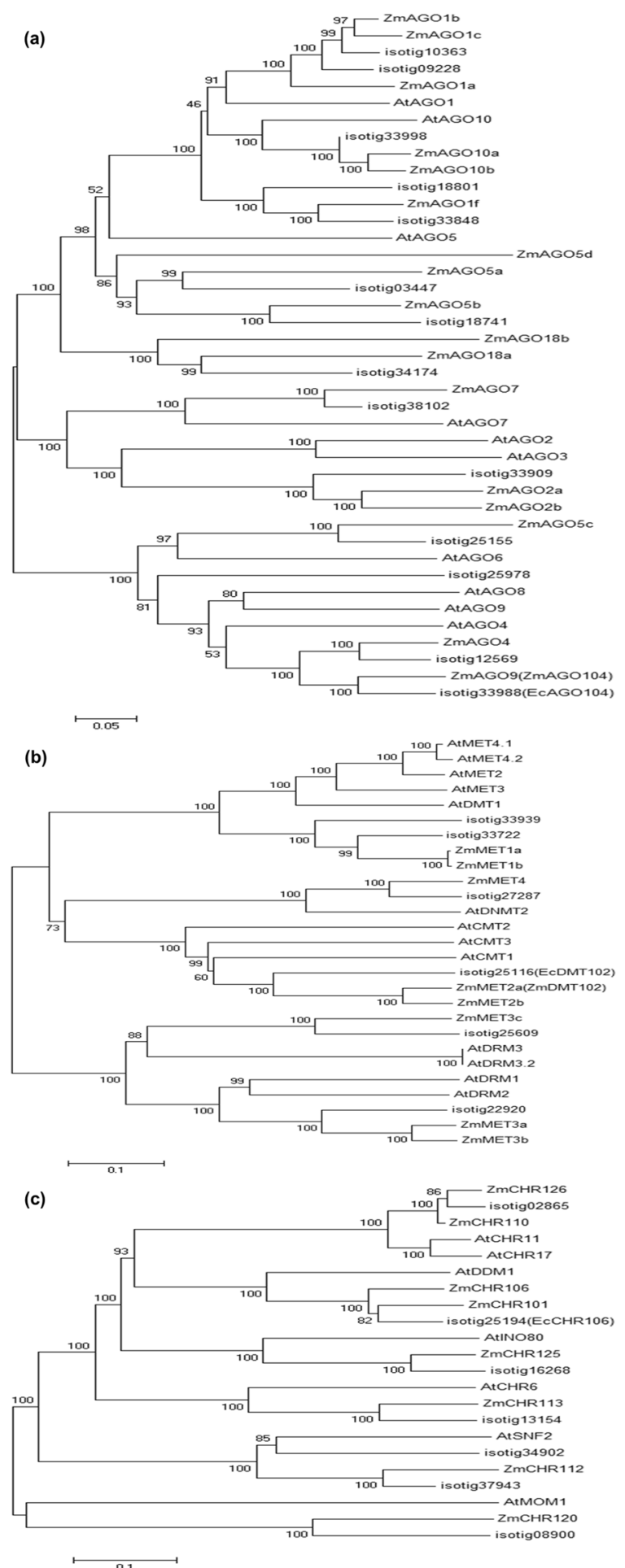

Figure 1. Phylogenetic trees of E. curvula RNA-dependent DNA methylation proteins in A. thaliana and Z. mays. (a) AGO, (b) DMT, and (c) CHR proteins from Arabidopsis and maize were retrieved from the The Arabidopsis Information Resource (TAIR) and the Maize Genetic and Genomic Database (MaizeGDB), respectively. The amino acid sequences of E. curvula were obtained from our floral reference transcriptomes. The unrooted neighbor-joining tree was constructed using MEGA v6.0.

was observed for EcAGO104 between OTA-S and Tanganyika samples during archesporial stages; however Tanganyika flowers expressed significantly more EcAGO104 than OTA-S flowers during gametogenesis (Fig. 3a). Furthermore, we detected higher expression levels of EcDMT102 in sexual plants across all developmental stages (Fig. 3b), whereas EcCHR106 transcripts were overrepresented in apomictic plants across both archesporial and gametophytic stages (Fig. 3c). 


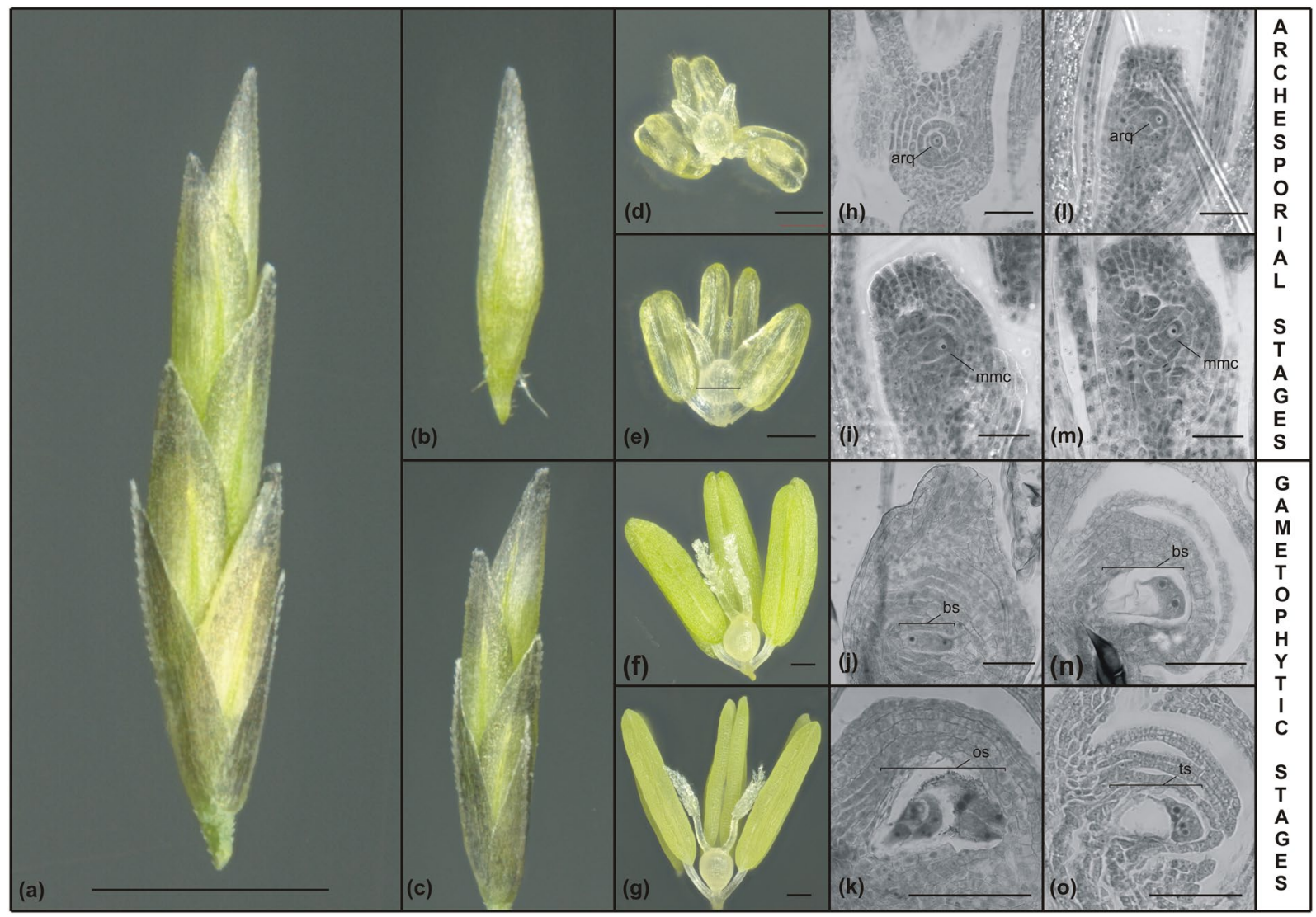

Figure 2. Reproductive calendar of sexual and apomictic individuals of Eragrostis curvula. (a) Whole spikelet with the anthecium containing all developmental stages. Scale bar: $1000 \mu \mathrm{m}$. (b,c) Stages used for total RNA extractions; (b) top of the spikelet with flowers containing ovules at archesporial and pre-meiotic stages, and (c) anthecium with pistils at gametophytic stages. (d-g) Ovaries and anthers at: (d) early archesporial stage, (e) late archesporial stage, (f) early gametophytic stage, and (g) late gametophytic stage. Scale bar: $100 \mu \mathrm{m}$. (h-k) Ovary sections from the sexual OTA genotype showing: (h) an archesporial cell, (i) a megaspore mother cell, (j) a binucleate embryo sac, and (k) a mature embryo sac with proliferating antipodal cells. Scale bar: $50 \mu \mathrm{m}$. (1-o) Ovary sections from Tanganyika apomicts showing embryo sac developmental course: (l) an archesporial cell, (m) a megaspore mother cell, (n) a binucleate embryo sac, and (o) a tetranucleate mature embryo sac. Scale bar: $50 \mu \mathrm{m}$. arq: arquespore, mmc: megaspore mother cell, bs: binucleate stage, oc: octonucleate stage, ts: tetranucleate stage.

Thus, although expression patterns can differ from those observed in other species (i. e. AGO104 and CHR106), we show here that the expression level of the selected candidate genes varies between apomictic and sexually reproducing plants, at least in specific developmental stages.

According to Olmedo-Monfil et al. ${ }^{11}$ most of AGO9-associated sRNAs are 24 nucleotides in length sRNAs and most 24-nucleotide sequences derived from TEs belonging to distinct families of retrotransposons: Gypsy (23\%) Athila (9.3\%), CACTA (5.5\%), and less frequently LINE or Mutator.

Thus, we mined the E. curvula reference transcriptome of Garbus et al. (manuscript under revision) and identified 267 putative LTR retrotransposons, including known targets of AGO9 (i.e. Athila retrotransposons ${ }^{20}$ ). Out of these, 63 were differentially represented between sexual and apomictic plants, but the differences were not conclusive. On the other hand, in a previous report on repetitive sequences expressed in cDNA libraries obtained from Eragrostis ${ }^{21}$, we showed differential expression for several members of the Gypsy retrotransposons family between inflorescences of sexual and apomictic genotypes. As already mentioned, our reference transcriptome covers all developmental stages. Since AGO9 activity was shown necessary to silence TEs in female gametes and their accessory cells ${ }^{11}$, this might provide an explanation for these observations. Alternatively, we show here that rather than downregulated, AGO9/AGO104 in Eragrostis apomicts is expressed in a different domain than in sexual plants. Therefore, such expression pattern could impact TE regulation in a different way that that reported for AGO9 mutant plants of Arabidopsis.

In situ hybridization analyses. Since plants lacking AGO9/AGO104 and DMT102 have reproductive phenotypes mimicking apomixis in Arabidopsis and maize ${ }^{11,13,14}$, in situ hybridization was conducted to determine the cell-specific expression patterns for these two genes in E. curvula reproductive organs (Figs 4 and 5). EcAGO104 is expressed at archesporial stages in ovules of both sexual and apomictic plants, but with contrasting distribution patterns. In sexual plants, strong but dispersed hybridization signals were observed in the nucellus 

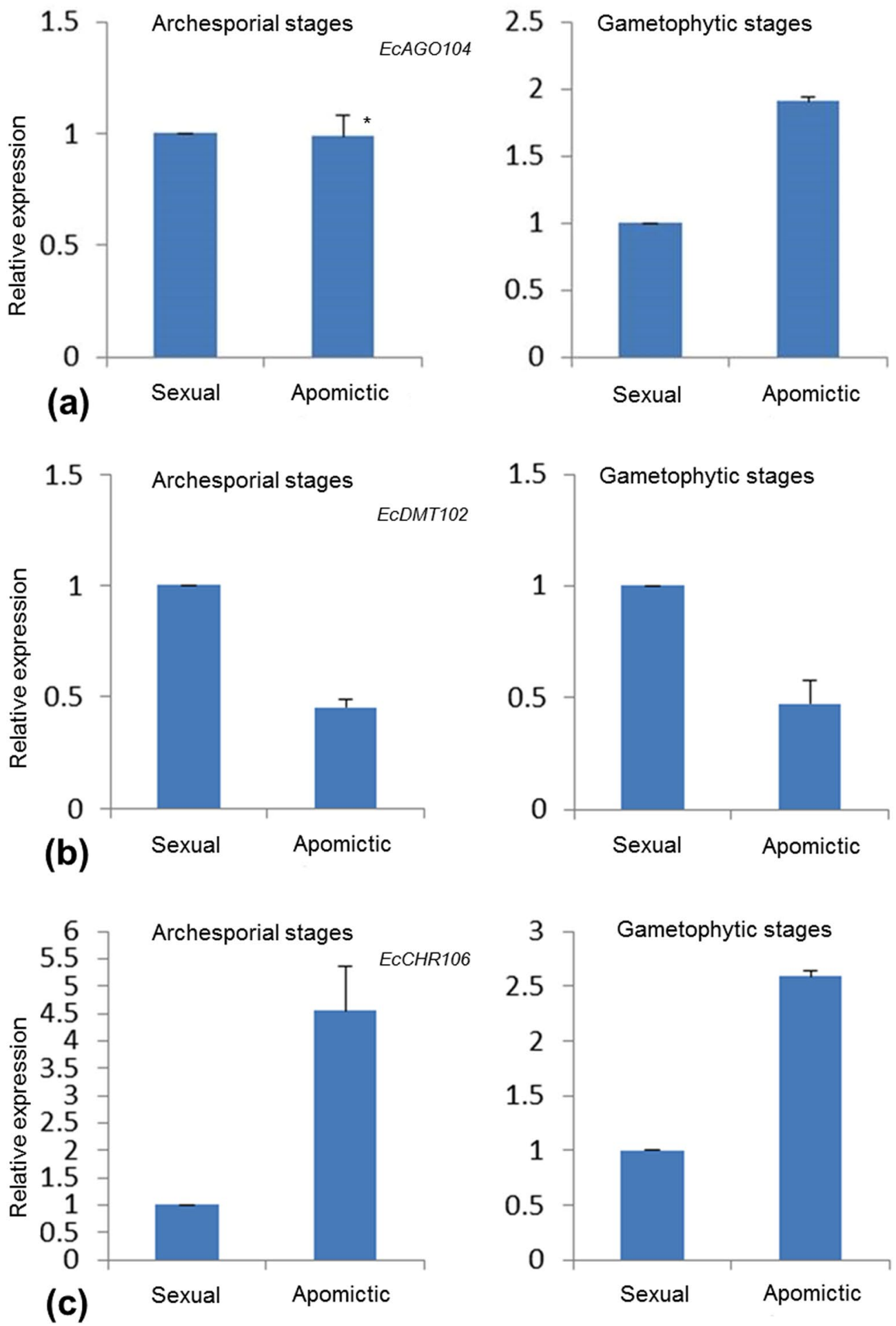

Figure 3. Quantitative RT-PCR of three RdDM genes during sexual and apomictic reproduction in E. curvula. Analyses were done using mRNA samples obtained from ovaries containing ovules at sporogenesis (archesporial stages) and megagametogenesis (gametophytic stages), Histograms show fold changes of expression levels for selected genes relative to control genes and error bars indicate the standard deviation for sample replicates. Differences between mean values were evaluated using Student's t-tests with paired samples $(\mathrm{P}<0.05)$. *Samples that were not validated

and the integuments, with no signal in the archesporial cell (Fig. 4a) and the MMC. By contrast, apomictic plants produced weak signals around the archesporial cell/MMC and a strong signal within the archesporial cell throughout development (Fig. 4e,f), although in the later stages, the signal seemed to be restricted only to the 


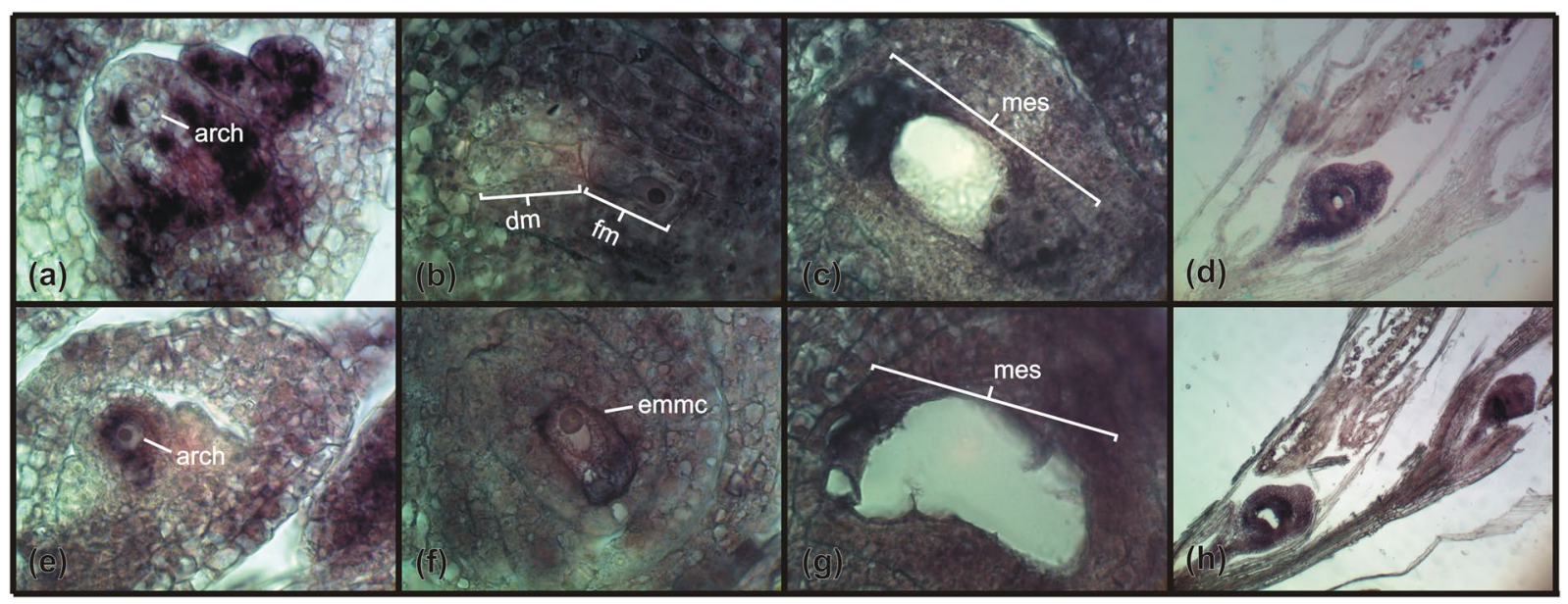

Figure 4. mRNA in situ hybridization of EcAGO104 during sexual and apomictic reproduction in E. curvula. (a-d) Ovaries from the sexual genotype hybridized with an EcAGO104 antisense probe showing: (a) an ovule primordium with strong, patchy signal in the nucellus and integuments and no signal in the archesporial cell; (b) signal restricted to the functional megaspore in a tetrad; (c) a mature embryo sac with all cells positive and no signal in the surrounding nucellar cells, and; (d) a general view of reproductive organs. (e-h) Ovaries from the apomictic genotype hybridized with an EcAGO104 antisense probe showing: (e) an apomictic ovule primordium with a strong signal detected in the archesporial cell only; (f) an elongated, unreduced MMC; (g) a mature embryo sac, and; (h) a general view of reproductive organs. Arch: archespore, dm: degenerated megaspores, fm: functional megaspore, mes: mature embryo sac, emmc: elongated megaspore mother cell.

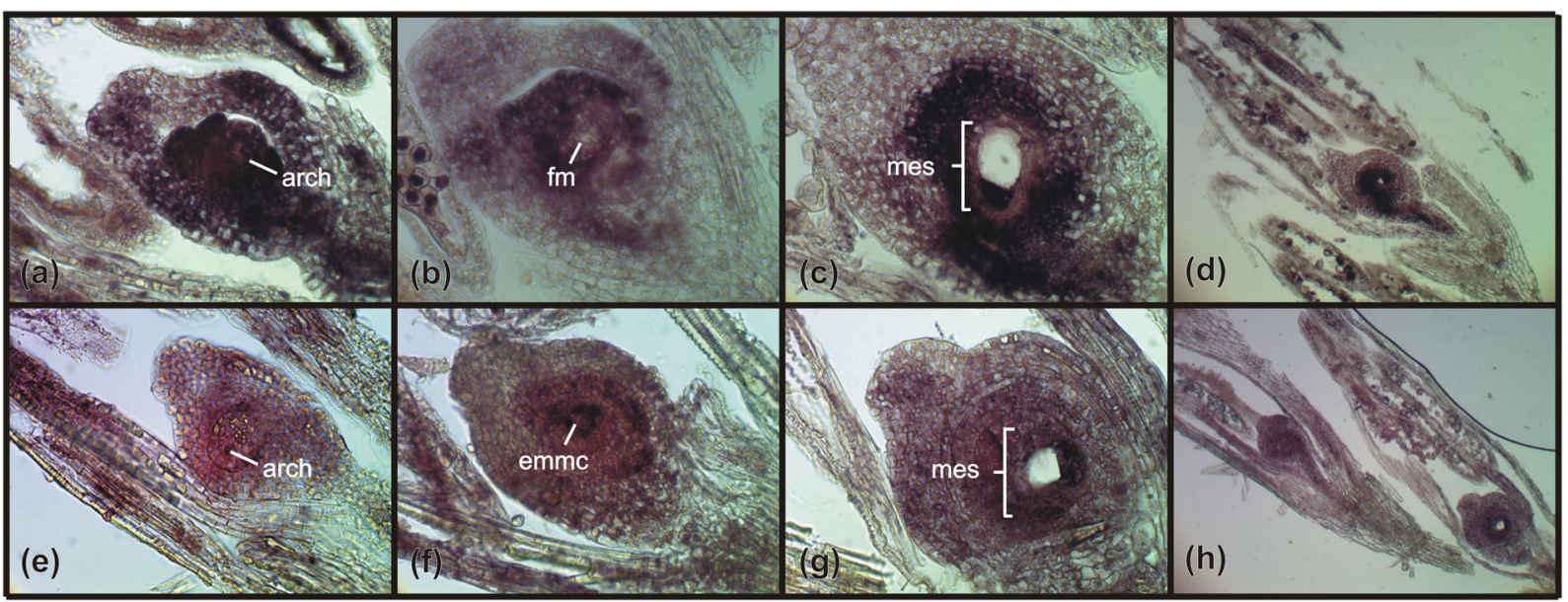

Figure 5. mRNA in situ hybridization of EcDMT102 during sexual and apomictic reproduction in E. curvula. (a-d) Ovaries from the sexual genotype hybridized with an EcDMT102 antisense probe showing strong signals in the nucellus and reproductive cells throughout development. (a) Young ovule primodium containing an archespore. (b,c) Ovules containing young and mature embryo sacs. (d) general view of reproductive organs. (e-h) Ovaries from the apomictic genotype hybridized with an EcDMT102 antisense probe showing weaker, but similarly distributed than in the sexual plants, signals. (e) Young ovule primodium containing an archespore. (f) Ovule containing an enlarged, unreduced MMC. (g) Ovules containing mature embryo sac. (h) General view of reproductive organs. arch: archespore, $\mathrm{dm}$ : degenerated megaspores, fm: functional megaspore, mes: mature embryo sac, emmc: elongated megaspore mother cell.

enlarged, unreduced MMC. Interestingly, EcAGO104 was first detected in the sexual germ line in functional surviving megaspores while micropylar megaspores produced no signal (Fig. 4b). Throughout female gametogenesis in both sexual and apomictic plants, a moderate expression of EcAGO104 was observed in the integuments and all cell types within the embryo sac (Fig. 4c,g). Finally, a strong signal was observed during male sporogenesis and gametogenesis in both somatic (tapetum) and reproductive cells (pollen mother cells, microspores) in both sexual and apomictic plants (Fig. 4d,h) indicating that EcAGO104 was differentially regulated during male and female reproductive developments between apomictic and sexual E. curvula plants.

In situ hybridization of EcDMT102 produced stronger signals in the ovules of sexual plants than apomictic plants across all female and male developmental stages (Fig. 5a-h), a result in agreement with both 454 
sequencing and RT-qPCR analyses. Nevertheless, both the spatial and temporal expression patterns remained similar between genotypes. In the early stages, while both the nucellus and integuments stained strongly, the signal was either weak or absent in the reproductive cells, i.e. the sexual MMC (Fig. 5a) and both the early and enlarged diplosporous MMC (Fig. 5e,f). During gametogenesis, expression was detected in nucellar cells and within the embryo sac, particularly at the micropylar end (Fig. 5b,c,g). Finally, the expression in the anthers followed a similar pattern, with stronger signal in sexual plants than in apomict plants (Fig. $5 \mathrm{~d}, \mathrm{~h}$ ).

Finally, hybridizations of ovules from both sexual and apomict plants collected at different developmental stages showed no signal for EcAGO104 sense probes whereas a moderate signal was observed for EcDMT102 (Supplemental Figure S1). This suggests antisense expression at a restrained level for EcDMT102. This phenomenon has been commonly observed for other apomixis-related candidates complexes, e.g. SOMATIC EMBRYOGENESIS RECEPTOR KINASE ${ }^{22}$ and ORIGIN RECOGNITION COMPLEX $3^{23}$, and it has been proposed to play a suppression role mediated by the formation sense-antisense mRNA complexes in apomict plants ${ }^{22,23}$.

\section{Discussion}

Here, we report expression analyses in the ovules of apomictic and sexual E. curvula individuals for three genes involved in DNA methylation pathways. Our results agree with previous findings in model plant species suggesting that RdDM misregulation in the ovule may promote apomictic-like phenotypes ${ }^{11-14}$; however, published expression data has also highlighted both similarities and differences between different diplosporous apomicts for the genes examined so far, i.e. two DMTs and CHR106/DDM $1^{14}$. In B. holboellii and T. dactyloides, CMT3/ DMT102/DMT105 was downregulated in apomictic plants ${ }^{14}$, in agreement with the results reported here for $E$. curvula. Contrastingly, CHR106/DDM1 was downregulated in T. dactyloides apomictic plants ${ }^{14}$, and showed no difference in $B$. holboellii apomictic and sexual plants, while we report here upregulation in $E$. curvula apomicts. In the present study, both quantitative and tissue-specific expression differences were detected for two genes (EcAGO104 and EcDMT102), changes which had already been associated with apomixis-like phenotypes in Arabidopsis and maize ${ }^{11,13,14}$.

AGO9/AGO104 proteins are specifically expressed in ovules of plants and are essential to specify cell fate, a function achieved by silencing heterochromatic sequences and, probably, genes ${ }^{11,13}$. However, mutant characterization in Arabidopsis and maize has revealed different functions during female reproduction, respectively repression of reproductive cell fate in the ovule and repression of somatic cell fate in the archespore. Therefore, loss-of-function in these two species generate different apomixis-like phenotypes reminiscent of apospory (Arabidopsis) and diplospory (maize). Our in-situ hybridization observations for EcAGO104 first suggest a similar function to that of maize AGO104 proteins. We detected signals in the female reproductive cell lineage from functional megaspores to mature embryo sacs in sexual plants and throughout all reproductive development in apomicts, including archesporial cells. This may indicates that ectopic EcAGO104 expression in the archespore could prevent entry into meiosis and promote gametophytic development. The weaker expression of EcAGO104 in the nucellus of apomictic plants before and during sporogenesis relative to that in sexually reproducing plants is an intriguing observation. Whether the phenomenon is required for triggering ectopic expression in the archespore remains unclear. One explanation, in agreement with the hypothesis for a non-cell autonomous, silencing signal spreading from sporophytic cells into reproductive cells during sporogenesis ${ }^{3}$, is to postulate an autoregulatory loop controlling EcAG0104 expression during sexual sporogenesis depending on mobile small RNAs generated in surrounding nucellar cells. Therefore, although our data also support a silencing pathway involving AGO9/ AGO104 operating in the nucellus of sexual plants and promoting meiosis, more analyses in both apomictic and sexual plants are required to decipher the mechanisms governing AGO9/AG0104 expression and to assess AGO9/ AGO104 function in plant ovules.

DMT102 is required for cytosine methylation at CNG sites, and it is likely involved in a maintenance function $^{24,25}$. In maize, DMT102 expression domain encompasses the reproductive cell and a few layers of nucellar cells ${ }^{14}$. This domain is conserved during early gametogenesis, while in mature ovules DMT102 expression becomes confined to the chalazal region of the embryo sac ${ }^{14}$. Similarly, in sexual E. curvula individuals, a strong signal was observed in the nucellus during early premeiosis; however, after gametogenesis, expression was detectable throughout the embryo sac (not only at the chalazal end).

In $d m t 102$ maize mutants, no apparent morphological nor reproductive phenotype, other than a high proportion of abnormally large pollen grains, was detected, and $d m t 102:: M u$ alleles are normally inherited both maternally and paternally ${ }^{14}$. In E. curvula diplosporous plants, we observed a significant level of EcDMT102 expression in the anthers, which might explain the absence of non-reduced pollen ${ }^{26}$. Maize $d m t 102$ homozygous mutant lines produce nearly full seed sets, indicating that most, if not all, female gametes are meiotically derived; however, at late gametophytic stages, large structures develop at the chalazal pole of the ovule, presumably after abnormal growth of antipodal cells ${ }^{14}$. These large chalazal cells remained arrested until the multicellular antipodal structure degenerates, suggesting that DMT102 might be involved in the maintenance of antipodal cell fate during late gametogenesis ${ }^{14}$. Interestingly, a downregulation of $E c D M T 102$ expression was observed in the nucellus of diplosporous E. curvula plants. These plants form embryo sacs lacking antipodal cells; therefore, it could be hypothesized that the natural downregulation of EcDMT102 in this species might be promoting the establishment of gametophytic specificities characteristic of apomictic individuals (ie, four-celled embryo sac), which differ from the canonical seven-celled embryo sacs observed in sexual individuals.

Contrary to observations indicating either down-regulation in maize-Tripsacum apomicts or steady-state expression in Boechera ${ }^{14}$, we found evidence for increased expression in Eragrostis apomicts of EcCHR106, an ortholog of the well-characterized Arabidopsis DDM1 gene. AtDDM1 encodes a nucleosome remodeling ATPase ${ }^{27}$, allowing DNA methyltransferases to access H1-containing heterochromatin ${ }^{28}$. In Arabidopsis, DDM1 and RdDM are both active in all DNA methylation contexts to silence TE, but with different efficiencies depending on chromatin organization. DDM1 is less efficient for short TEs, usually found nearby genes (ie, euchromatic regions) 
and, therefore, silencing relies more on RdDM. Oppositely, silencing in heterochromatic regions is more effective through DDM1 than $\mathrm{RdDM}^{28}$. Our data indicate that DDM1 function is enhanced, or at least preserved, throughout ovule development in Eragrostis apomicts while RdDM is inactivated. This suggests that chromatin states nearby and within genes could be more permissive in apomict ovules and, therefore, supports the notion that $\mathrm{RdDM}$ is involved in the establishment of gene expression patterns governing the switch from sexuality to diplosporous apomixis in plants. Recent progress in understanding DNA methylation patterns in plants has revealed differences in mechanisms and functions for both canonical and non-canonical RdDM pathways across species $^{29,30}$. These specificities might have sustained the emergence of diversified reproductive behaviors, including the differences observed among apomictic developments.

\section{Materials and Methods}

Biological materials and resources. Two tetraploid $(2 \mathrm{n}=4 \mathrm{x}=40)$ accessions of weeping lovegrass provided by the United States Department of Agriculture (USDA) were used: the apomictic Tanganyika (PI234217) and the sexual OTA-S (PI574506) genotypes. Plants were grown in a glasshouse at $25^{\circ} \mathrm{C}$ under natural light conditions. For both genotypes, a reproductive calendar was compiled based on anatomical (spikelets size and morphology) and histological observations. This calendar encompasses four developmental stages from early archespores to mature female gametophytes (Fig. 2).

FLX Roche 454 sequencing. Spikelets with basal flowers at the beginning of anthesis, representing all developmental stages, were collected and total RNA was extracted using a NucleoSpin ${ }^{\circledR}$ miRNA kit (Machery-Nagel, Düren, Germany). Each genotype was represented by two biological replicates. The samples were sequenced using the 454 GS FLX + Roche method at INDEAR (Instituto de Agrobiotecnología de Rosario, Santa Fe, Argentina). Raw reads were deposited in the Sequence Reads Archive (SRA) database at NCBI as BioProject 358210 "Floral transcriptome of sexual and apomictic Eragrostis curvula" including Biosamples SAMN06167423 (reads from OTA-S) and SAMN06167424 (reads from Tanganyika). Curated raw sequences were assembled using Newbler Assembler software v2.6 (Roche, Indianapolis, IN, USA).

Differential expression between two conditions was analyzed using the EdgeR package ${ }^{31}$ and P-values adjusted using the Benjamini and Hochberg method ${ }^{32}$ to control the false discovery rate. A corrected P-value of 0.01 and log2 (fold-change) of 1 were used as thresholds for significant differential expression.

Phylogenetic and sequence analyses. Arabidopsis and maize protein sequences for AtAGO9/ZmAGO104, AtDDM1/ZmCHR106, and AtCMT3/ZmDMT102 were retrieved, respectively, from The Arabidopsis Information Resource (TAIR) and the Maize Genetic and Genomic Database (MaizeGDB), and used to query the 454 RNA libraries using BLASTP. Gene families were aligned using a BLOSUM30 matrix ${ }^{33}$ and ClustalW in pairwise alignments (open gap penalty: 10; gap extend penalty: 0.1) and multiple alignments (gap extend penalty: 0.2; delay divergent setting: 30\%) were generated. A phylogenetic reconstruction was conducted using a neighbor-joining method in MEGA v6.0 $0^{34}$. Unrooted consensus trees were obtained from 1000 bootstrap replicates.

RT-qPCR experiments. Flowers from OTA-S (sexual plant) and Tanganyika (apomictic plant) were collected and divided in both archesporial and gametophytic stages (Fig. 2). Total RNA was extracted as described above. The RNA was reverse-transcribed using the ImProm-II ${ }^{\mathrm{TM}}$ Reverse Transcription System (Promega, Madison, WI, USA). Real-time PCR reactions were conducted as previously ${ }^{35}$, using primers designed with the SciTools software (http://www.idtdna.com) from Integrated DNA Technologies (Coralville, IA, USA) (Supplementary Table S1). UBIQUITIN CONJUGATING ENZYME (UBICE) and GLUCOSE-6-PHOSPHATE DEHYDROGENASE (GPDH) were used as reference genes, after validating their steady-state expression patterns throughout reproductive development in the plants using the gNorm software ${ }^{36}$ (data not shown). Non-template controls were incorporated into all assays. For each sample, two biological replicates were processed in triplicate. Amplifications were performed in a Rotor-Gene 6000 (Corbett Life Sciences) using the following conditions: $2 \mathrm{~min}$ at $94^{\circ} \mathrm{C}$, followed by 40 cycles of $15 \mathrm{~s}$ at $95^{\circ} \mathrm{C}, 20 \mathrm{~s}$ at $55^{\circ} \mathrm{C}$, and $30 \mathrm{~s}$ at $72^{\circ} \mathrm{C}$. A melting curve was produced at the end of each reaction to check amplicon specificity. Amplification efficiency was calculated with Rotor-Gene 6000 Series Software 1.7. Relative gene expression was assessed using the $2^{-\Lambda \Lambda \mathrm{CT}}$ method $^{37}$. Differences between mean values were evaluated by Student's t-tests. $P$ values $<0.05$ were considered significant.

In situ hybridization experiments. Sense and antisense DIG-labelled RNA probes were produced for the $E c A G O 104$ and EcDMT102 genes. Hybridizations were performed for the ovaries of plants at different developmental stages, following the protocol previously described by Laspina et al. ${ }^{38}$ with minor modifications. Briefly, inflorescences at pre-anthesis were fixed in $4 \%$ paraformaldehyde $/ 0.25 \%$ glutaraldehyde in $0.01 \mathrm{M}$ phosphate buffer $\mathrm{pH} 7.2$, dehydrated in an ethanol-xylol series and embedded in paraffin. Spikelets were cut into 7-10 $\mu \mathrm{m}$ thin sections and placed onto slides treated with $100 \mu \mathrm{g} / \mathrm{ml}$ poly-l-lysine. Paraffin was removed using an ethanol-xylol series. Plasmids containing the selected clones were linearized using the restriction enzymes NcoI or SalI (Promega). The amplicons 577-nt and 555-nt long originated from EcAGO104 and EcDMT102 were used as probes in hybridization analysis (Supplementary Table S1). Probes were labeled using the Roche Dig RNA Labeling kit, following the manufacturer's instructions. The probes were hydrolyzed to $150-200 \mathrm{bp}$ fragments. Pre-hybridization was carried out in $0.05 \mathrm{M}$ Tris- $\mathrm{HCl} \mathrm{pH} 7.5$ buffer containing $1 \mu \mathrm{g} / \mathrm{mL}$ proteinase $\mathrm{K}$ in a humid chamber at $37^{\circ} \mathrm{C}$ for $10 \mathrm{~min}$. Hybridization was carried out overnight in a humid chamber at $37^{\circ} \mathrm{C}$, in $10 \mathrm{mM}$ Tris- $\mathrm{HCl}$ pH 7.5 buffer containing $300 \mathrm{mM} \mathrm{NaCl}, 50 \%$ formamide (deionized), 1 mM EDTA pH 8, 1x Denhardt, $10 \%$ dextransulphate, $600 \mathrm{ng} / \mathrm{ml}$ total RNA and $60 \mathrm{ng}$ of the corresponding probe. Detection was performed following the Roche Dig Detection kit instructions, using anti-DIG AP and NBT/BCIP. Three independent experiments, each involving at least 20 spikelets, were conducted per genotype. 


\section{References}

1. Nogler, G. Gametophytic apomixis in Embryology of Angiosperms (ed. Johri, B. M.) 475-518 (Springer, 1984).

2. Ozias-Akins, P. \& van Dijk, P. Mendelian genetics of apomixis in plants. Annu Rev Genet 41, 509-537 (2007).

3. Grimanelli, D. Epigenetic regulation of reproductive development and the emergence of apomixis in angiosperms. Curr Opin Plant Biol 15, 57-62 (2012)

4. Reiser, L. \& Fischer, R. L. The ovule and the embryo sac. Plant Cell 5, 1291-1301 (1993).

5. Bicknell, R. A. \& Koltunow, A. M. Understanding apomixis: recent advances and remaining conundrums. Plant Cell 16, S228-S245 (2004).

6. Hand, M. L. \& Koltunow, A. M. G. The genetic control of apomixis: asexual seed formation. Genetics 197, 441-450 (2014).

7. Schmidt, A. et al. Apomictic and sexual germline development differ with respect to cell cycle, transcriptional, hormonal and epigenetic regulation. PLoS Genet 10, e1004476 (2014).

8. Galla, G., Vogel, H., Sharbel, T. F. \& Barcaccia, G. De novo sequencing of the Hypericum perforatum L. flower transcriptome to identify potential genes that are related to plant reproduction sensu lato. BMC Genomics 16, 254 (2015).

9. Galla, G. et al. Pistil transcriptome analysis to disclose genes and gene products related to aposporous apomixis in Hypericum perforatum L. Front Plant Sci 8, 79 (2017).

10. Rabiger, D. S. et al. Generation of an integrated Hieracium genomic and transcriptomic resource enables exploration of small RNA pathways during apomixis initiation. BMC Biol 14, 86 (2016).

11. Olmedo-Monfil, V. et al. Control of female gamete formation by a small RNA pathway in Arabidopsis. Nature 464, 628-632 (2010).

12. Hernández-Lagana, E., Rodríguez-Leal, D., Lúa, J. \& Vielle-Calzada, J.-P. A multigenic network of ARGONAUTE4 clade members controls early megaspore formation in Arabidopsis. Genetics 204, 1045-1056 (2016).

13. Singh, M. et al. Production of viable gametes without meiosis in maize deficient for an ARGONAUTE protein. Plant Cell 23, 443-458 (2011).

14. García-Aguilar, M., Michaud, C., Leblanc, O. \& Grimanelli, D. Inactivation of a DNA methylation pathway in maize reproductive organs results in apomixis-like phenotypes. Plant Cell 22, 3249-3267 (2010).

15. Poverene, M. \& Voigt, P. Isozyme variation and germplasm relationships in the Eragrostis curvula complex. Biochem Syst Ecol 25, 21-32 (1997).

16. Voigt, P., Rethman, N. \& Poverene, M. Lovegrasses in Warm-Season (C4) Grasses, Agron Monogr 45 (ed. Moser, L. E., Burson, B. L. \& Sollenberger, L. E.) 1027-1056 (ASA, CSSA, SSSA, 2004).

17. Garbus, I. et al. De novo transcriptome sequencing and assembly from apomictic and sexual Eragrostis curvula genotypes. PLOS ONE, doi:10.1371/journal.pone.0185595 (2017).

18. Zhai, L. et al. Identification and characterization of Argonaute gene family and meiosis-enriched Argonaute during sporogenesis in maize. J Integr Plant Biol 56, 1042-1052 (2014).

19. Qian, Y. X., Xi, Y. L., Cheng, B. J. \& Zhu, S. W. Genome-wide identification and expression profiling of DNA methyltransferase gene family in maize. Plant Cell Rep 33, 1661-1672 (2014).

20. Durán-Figueroa, N. \& Vielle-Calzada, J. P. ARGONAUTE9-dependent silencing of transposable elements in pericentromeric regions of Arabidopsis. Plant Signal Behav 5, 1476-1479 (2010).

21. Romero, J. et al. Repetitive sequences in Eragrostis curvula cDNA EST libraries obtained from genotypes with different ploidy. Biol plantarum 60, 55-67 (2016).

22. Podio, M. et al. Characterization and expression analysis of SOMATIC EMBRYOGENESIS RECEPTOR KINASE (SERK) genes in sexual and apomictic Paspalum notatum. Plant mol biol 84, 479-495 (2014).

23. Siena, L. A. et al. An apomixis-linked ORC3-like pseudogene is associated with silencing of its functional homolog in apomictic Paspalum simplex. J exp bot 67, 1965-1978 (2016).

24. Papa, C. M., Springer, N. M., Muszynski, M. G., Meeley, R. \& Kaeppler, S. M. Maize chromomethylase Zea Methyltransferase2 is required for CpNpG methylation. Plant Cell 13, 1919-1928 (2001).

25. Makarevitch, I. et al. Natural variation for alleles under epigenetic control by the maize chromomethylase zmet2. Genetics $\mathbf{1 7 7}$, 749-760 (2007).

26. Voigt, P. \& Bashaw, E. Apomixis and sexuality in Eragrostis curvula. Crop Sci 12, 843-847 (1972)

27. Jeddeloh, J. A., Stokes, T. L. \& Richards, E. J. Maintenance of genomic methylation requires a SW12/SNF2-like protein. Nat Genet 22, 94-97 (1999).

28. Zemach, A. et al. The Arabidopsis nucleosome remodeler DDM1 allows DNA methyltransferases to access H1-containing heterochromatin. Cell 153, 193-205 (2013).

29. Ma, L. et al. Angiosperms are unique among land plant lineages in the occurrence of key genes in the RNA-directed DNA methylation (RdDM) pathway. Genome Biol Evol 7, 2648-2662 (2015).

30. Xie, M. \& Yu, B. siRNA-directed DNA methylation in plants. Curr Genomics 16, 23-31 (2015).

31. Robinson, M. D., McCarthy, D. J. \& Smyth, G. K. edgeR: a Bioconductor package for differential expression analysis of digital gene expression data. Bioinformatics 26, 1 (2010).

32. Benjamini, Y. \& Hochberg, Y. Controlling the false discovery rate: a practical and powerful approach to multiple testing. $J$ Roy Stat Soc B 57, 289-300 (1995).

33. Henikoff, S. \& Henikoff, J. G. Amino acid substitution matrices from protein blocks. Proc Natl Acad Sci USA 89, 10915-10919 (1992).

34. Tamura, K., Stecher, G., Peterson, D., Filipski, A. \& Kumar, S. MEGA6: molecular evolutionary genetics analysis version 6.0. Mol Biol Evol 30, 2725-2729 (2013).

35. Selva, J. P., Pessino, S., Meier, M. \& Echenique, V. Identification of candidate genes related to polyploidy and/or apomixis in Eragrostis curvula. Am J Plant Sci 3, 403-416 (2012).

36. Vandesompele, J. et al. Accurate normalization of real-time quantitative RT-PCR data by geometric averaging of multiple internal control genes. Genome Biol 3, RESEARCH0034 (2002).

37. Livak, K. J. \& Schmittgen, T. D. Analysis of relative gene expression data using real-time quantitative PCR and the 2 (-Delta Delta C(T)) Method. Methods 25, 402-408 (2001).

38. Laspina, N. V. et al. Gene expression analysis at the onset of aposporous apomixis in Paspalum notatum. Plant Mol Biol 67, 615-628 (2008).

\section{Acknowledgements}

This project has received funding from the European Union's Horizon 2020 Research and Innovation Programme under the Marie Skłodowska-Curie Grant Agreement No 645674; Agencia Nacional de Promoción Científica y Tecnológica (ANPCyT), Argentina, Projects PICT Raíces 2014 1243; PICT 2013-2029, PICT 2011-1269, PICT2014-1080; Consejo Nacional de Investigaciones Científicas y Técnicas (CONICET), Argentina, Project: PIP 11220110100767; PGI UNS Project 24A199. J.M. Rodrigo is a CONICET postdoctoral fellow. J. Romero was a CONICET professional technician. J.P. Selva, L. Siena, I. Garbus, D. Zappacosta, J.P.A. Ortiz, S. Pessino and V. Echenique are CONICET research staff members. 


\section{Author Contributions}

J.P.S. conceived and designed the experiments, analyzed the data and wrote the first draft of the manuscript. J.M.R., L.S. and D.Z. performed the in situ hybridization analysis. J.P.A.O. contributed to the analysis and interpretation of qRT-PCR and the in situ hybridization. J.R.R. and I.G. performed the sequence analyses. S.C.P., O.L. and V.E. conceived part of the experimental design, and contributed to data interpretation and manuscript writing. All authors reviewed and approved the final manuscript.

\section{Additional Information}

Supplementary information accompanies this paper at https://doi.org/10.1038/s41598-017-14898-5.

Competing Interests: The authors declare that they have no competing interests.

Publisher's note: Springer Nature remains neutral with regard to jurisdictional claims in published maps and institutional affiliations.

(c) Open Access This article is licensed under a Creative Commons Attribution 4.0 International License, which permits use, sharing, adaptation, distribution and reproduction in any medium or format, as long as you give appropriate credit to the original author(s) and the source, provide a link to the Creative Commons license, and indicate if changes were made. The images or other third party material in this article are included in the article's Creative Commons license, unless indicated otherwise in a credit line to the material. If material is not included in the article's Creative Commons license and your intended use is not permitted by statutory regulation or exceeds the permitted use, you will need to obtain permission directly from the copyright holder. To view a copy of this license, visit http://creativecommons.org/licenses/by/4.0/.

(c) The Author(s) 2017 